J. Lake Sci.(湖泊科学), 2021, 33(2): 336-348

DOI 10. 18307/2021. 0202

(c) 2021 by Journal of Lake Sciences

\title{
稻田氮磷径流流失模型研究进展”
}

\author{
黄微尘 ${ }^{1,2}$, 周 丰 $^{1,2 * *}$, 梁 浩 $^{3}$, 陈 䂞 $^{4}$ \\ (1: 北京大学城市与环境学院, 北京 100871) \\ ( 2 : 北京大学地表过程分析与模拟教育部重点实验室, 北京 100871) \\ (3: 河海大学农业科学与工程学院, 南京 210098) \\ (4: 北京师范大学环境学院, 北京 100875 )
}

摘 要: 水稻是全球最重要的粮食作物, 但种植过程中不合理的水肥管理引起了稻田氮磷径流流失, 对邻近水体造成污 染风险. 稻田氮磷径流流失模型是理解区域水体污染排放时空特征和评估水污染风险的有效手段. 论文阐述了稻田氮磷 径流流失的发生机制和关键过程, 梳理了统计经验模型、物理机理模型、水文过程模型和生态系统模型的研发历程. 结果 表明, 当前模型缺乏水一土一气界面过程的有效参数化, 基于高分辨率、多年多站点、全通量的模型参数的合理化, 以及 区域尺度农艺管理 (种植制度、施肥制度、灌排制度) 和田一沟一塘联合调控措施 (分布、规模、运行规则) 的模块化, 造成了 稻田氮磷径流流失的不确定性. 同时, 提出了面向稻田氮磷循环的高分辨率全通量观测网络建设、农艺管理与调控措施 的区域数据集研发、模型开发与先进技术手段的集成与融合、稻田氮一磷一水耦合的多模型集合等, 为稻田氮磷径流流失 模型的改进、农艺管理措施的优化以及气候智能型稻田的建设提供科学依据.

关键词: 稻田; 氮循环; 磷循环; 径流流失; 过程模型

\section{Research progress on nitrogen and phosphorus runoff loss models for rice paddy ${ }^{*}$}

\author{
Huang Weichen ${ }^{1,2}$, Zhou Feng ${ }^{1,2 * *}$, Liang $\mathrm{Hao}^{3} \&{\text { Chen } \mathrm{Lei}^{4}}^{4}$ \\ (1: College of Urban and Environmental Sciences, Peking University, Beijing 100871, P.R.China) \\ (2: Laboratory for Earth Surface Process, Peking University, Beijing 100871, P.R.China) \\ (3: College of Agricultural Science and Engineering, Hohai University, Nanjing 210098, P.R.China) \\ (4: School of Environment, Beijing Normal University, Beijing 100875, P.R.China)
}

Abstract: Rice is the most significant food crop in the world, but nitrogen and phosphorus runoff loss, a risk of pollution to adja-
cent water bodies, would occur during cultivation by unreasonable water and fertilizer management. Nitrogen and phosphorus runoff
loss model for rice paddies is an effective way, in addition to assessing the risk of water pollution, to understand the spatial and
temporal characteristics of regional water pollution discharge. Here, the authors expound the occurrence mechanism and key proces-
ses of nitrogen and phosphorus runoff loss in rice paddies, and then systematically reviews the research and development history of
statistical empirical model, physical mechanism model, hydrological process model and ecosystem model. The authors point out that
current models are limited by the uncertainty of nitrogen and phosphorus runoff losses in paddy fields, due to deficiency in the ef-
fective parameterization of the water-soil-air interface process, rationalization of model parameters based on high resolution, multi-
year, multi-site and full flux, as well as regional-scale agricultural management (planting, fertilization, irrigation and drainage sys-
tem) and the modularization of the joint control measures ( distribution, scale, operation rules) of paddy-ditch-pond. Finally, the
authors propose outlook on the establishment of high-resolution and full flux observation network for rice paddies' nitrogen and phos-
phorus cycles, regional data set research and development of agricultural management and control measures, integration and fusion
of model development and advanced technological means, and multi-model integration of nitrogen-phosphorus-water coupling in rice
paddies. Achieving these prospects will provide scientific support for the improvement of nitrogen and phosphorus runoff models for

* 2020-05-06 收稿;2020-08-01 收修改稿.

国家重点研发计划项目 (2016YFD0800501) 和国家自然科学基金项目 (41977082) 联合资助.

** 通信作者;E-mail:zhouf@ pku.edu.cn. 
a rice paddy, the optimization of agricultural management measures, and the construction of climate-smart rice paddies

Keywords: Rice paddies; nitrogen cycle; phosphorus cycle; runoff loss; process model

稻米是全球超过一半人口的主食 ${ }^{[1]}$, 稻田分布在全球 120 多个国家,但 $90 \%$ 的播种面积集中在东亚、南 亚和东南亚 ${ }^{[2]} .2017$ 年, 稻田消耗了全球 50\% 的灌溉量和 $14 \%$ 的化肥施用量 ${ }^{[3]}$, 该比例在中国更高, 超过 $60 \%$ 和 $20 \%{ }^{[2]}$. 这种粗放的水肥管理, 再加上气候变化, 造成了过量的氮磷径流流失, 严重威胁区域水安 全 ${ }^{[4]}$. 有研究表明, 中国稻田氮磷径流流失自 1990 年以来分别增加了 $46 \%$ 和 $30 \%{ }^{[5]}$, 且未来这一风险将进 一步提高 ${ }^{[6]}$. 然而, 水稻品种类型繁多、种植制度多样、水肥管理迥异, 稻田氮磷径流流失过程十分复杂, 未 来时空格局及其生态环境效应还存在较大的不确定性.

稻田氮磷径流流失的影响因素众多,包括降水特征(强度、雨型、持续时间等) ${ }^{[7]}$ 、土壤理化性质 ${ }^{[8]}$ 、农田 管理措施 ${ }^{[9]}$ 、肥料管理 (强度、类型、方法和时间 $)^{[10]}$ 、水分管理 (灌溉方式、田埂排水高度 $)^{[11]}$ 等. 同时, 稻田 氮磷径流流失过程还与土壤养分循环的其他过程密切相关 (如作物吸收、淋溶、挥发等), 完全通过观测手段 来解析稻田氮磷径流流失的区域规律是不现实的. 因此, 提高稻田氮磷径流流失过程模拟能力, 是优化稻田 水肥管理和实现气候智能型稻田的关键手段.

自 1950s 以来, 研究者陆续开发了适用于稻田氮磷径流流失的多种模型, 并利用不同区域、尺度的观测 和控制试验进行了模型校准和验证. 这些模型大体上分为 4 类,包括统计经验模型、物理机理模型、水文过 程模型和生态系统过程模型, 从简单的因果分析到复杂的机理探索, 从单一模块的开发到多模块的耦合, 已 经具备了田块尺度、单一场次降雨到区域尺度连续降雨的多情景预测功能. 目前, 已有不少相关方面的综述 文章,如梳理 SWAT( soil and water assessment tool)、DNDC(denitrification-decomposition)等某一个具体模型的 发展和应用历程 ${ }^{[12-13]}$,或是聚焦面源污染、水文过程、作物生长等某一类模型的方法研发和改进 ${ }^{[14-16]}$,也有 着眼于降雨-径流、污染物迁移转化、氮磷循环等具体的过程机理及影响因素 ${ }^{[17-19]}$. 模型开发和应用中具体 的环节, 如数据获取、参数率定、不确定性分析 ${ }^{[20-22]}$, 也是综述文章的一个重要方面. 然而, 系统总结稻田氮 磷径流流失模型研发历程, 尤其是其中降雨引起的释放和输移等过程以及农艺管理措施等方面的综述仍较 少,也尚未系统归纳模型的关键问题或技术难点.

鉴于此, 本文将系统阐释稻田氮磷径流流失发生机制, 梳理国内外稻田径流流失 4 类模型的研发历程, 对比分析主流模型的性能特征, 归纳模型在发生机制参数化、模型参数合理化以及农艺管理措施模块化等 关键问题,在此基础上提出模型研究展望,为稻田氮磷径流流失模型的改进和应用提供科学依据.

\section{1 稻田氮磷径流流失发生机制}

与旱地不同的是, 稻田在水稻生长季会经历频繁的干湿交替过程, 为水稻和土壤微生物提供了独特的 生境 ${ }^{[23]}$, 发生了作物吸收、肥料水解、有机质矿化、养分溶解态和吸附态互相转化等过程. 由于特殊的淹水 环境, 稻田土壤呈现还原性, 有利于反硝化作用的产生. 同时, 田面水溶解氧较高, 水稻茎秆通气较好, 使得 水土界面又形成氧化层,促进硝化作用的发生 ${ }^{[19]}$. 因此,水稻种植具有与稻田环境相应、区别于旱地的田间 措施、水肥管理制度和种植方式 ${ }^{[24]}$.

稻田氮磷径流流失发生机制总结如图 1. 稻田的氮磷输人主要包括干湿沉降、灌溉、施肥和生物固氮. 当 发生降雨时, 雨滴携带氮磷进人田面水中, 改变田面水水位及氮磷浓度 ${ }^{[5,25]}$. 与旱地直接产生土壤浌蚀和颗 粒分离不同, 稻田田面水层对于降雨的冲击力还具有缓冲作用, 降低了滴溅侵蚀能力 ${ }^{[26]}$. 雨水经过水稻冠 层截流之后, 剩余的雨滴能量在田面水表层转化成势能, 产生间歇性的压力扰动, 造成水土界面土壤孔隙水 的释放 ${ }^{[7]}$. 土壤中存在氮磷的垂向扩散, 同时由于重力作用, 土壤表层溶质也会向下渗透. 随着雨量的增大, 土壤淋溶损失逐渐增大至饱和, 当降水量大于渗透率, 田面水位开始抬升并超过田埂, 产生地表径流 ${ }^{[27]}$. 孔 隙水、田面水和降雨灌溉水混合后形成水平推流, 经过田间的输移过程, 以溶解态氮素和吸附态磷素为主到 达排水口,排出稻田形成地表径流流失,这一过程中部分氮磷又通过吸附解析作用再次沉淀.

由于暴雨事件往往具有瞬时性和短暂性, 稻田氮磷径流流失对于初始条件敏感性较高. 初始田面水位、 田面水浓度、土壤浓度等会显著影响到氮磷流失量 ${ }^{[7]}$. 因此,施肥量、施肥方式以及灌排制度通过改变稻田 
初始水量和养分含量影响流失过程. 其中, 肥料在施用之后, 短时间内能显著提高田面水氮磷浓度,增加流 失风险 ${ }^{[28]}$. 同时土壤中氮磷三态的相互转化、作物吸收、蒸散发、氨挥发、反硝化造成的气体排放、干沉降、 淋溶、侧渗等过程都会通过间接途径参与整个稻田系统的径流流失. 由此可见, 有效模拟稻田径流流失, 需 要准确量化径流流失过程、稻田初始条件和水肥管理制度.

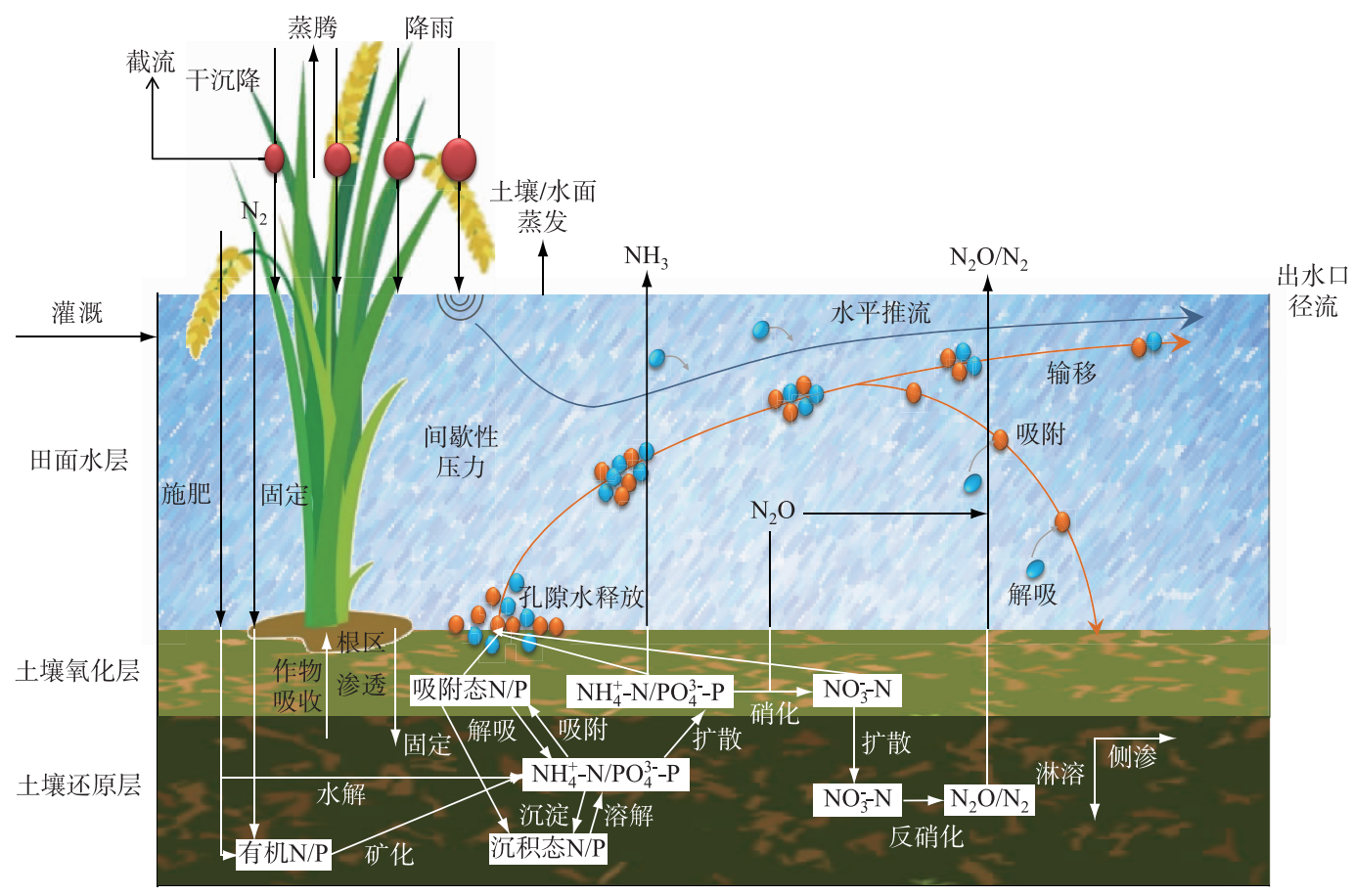

图 1 稻田氮磷径流流失发生机制与主要过程

Fig.1 Occurrence mechanism and key processes of nitrogen and phosphorus runoff losses from rice paddies

\section{2 模型研发历程}

\section{1 统计经验模型}

统计经验模型是基于观测数据, 利用因果分析和统计分析方法, 建立的不同形态氮磷流失通量与降雨 量、施肥量、灌溉量等因素的响应方程, 但难以考虑稻田复杂的水文和氮磷迁移转化过程 (图 2). 例如, SCS 模型 ( soil conservation service) ${ }^{[29]}$ 根据土壤和降雨因素来确定径流总量,包含一个代表土壤通透性的重要综 合参数 $C N$ ( curve number), 可适应多种土壤类型和土地利用类型下的径流量模拟. 随后, 研究者通过实验手 段调整不同稻区的具体数值 ${ }^{[30-31]}$. Lian 等 ${ }^{[32]}$ 搜集了 55 个研究地点的大量降雨径流数据, 建立了 $C N-C h i n a$ 查找表, 提供了适用于中国的参考指南. 另外, 输出系数模型 ${ }^{[33]}$ 是根据田间实验确定污染物输出系数, 应用 于稻田年尺度氮磷径流流失负荷估算. SPARROW 模型 (spatially referenced regressions on watershed attributes $)^{[34]}$ 将统计学方法应用于污染物的质量传输过程, 为大尺度应用提供了一种体现区域差异的统计方法.

由于统计经验模型只考虑输人和输出之间的关系, 不涉及具体的过程和机理, 对数据质量要求较低, 适 合于大尺度、年尺度的氮磷径流流失负荷估算. 例如, 我国全国第一次污染源普查就采用输出系数法对全国 尺度稻田的污染物流失系数进行核算. Hou 等 ${ }^{[25]}$ 考虑施氮强度、气候、灌溉量和土壤属性等环境因子对氮流 失率的影响, 建立了农田总氮径流流失量与施氮强度之间的非线性响应模式 (附表 I 和 II ), 更新了中国第 一次污染普查的结果. 同时, 统计经验模型也多用来探索多输人变量之间的相互关系, 来获取稻田氮磷径流 流失过程的关键控制因子, 识别有针对性的减排措施 ${ }^{[5,33]}$. 当数据存在缺漏时, 还可应用统计模型建立回归 关系补全数据序列. 由于其操作简便, 所需数据资料少, 常纳人其他模型的模块中, 或结合简单的机理模型 
提高其区域模拟的能力 ${ }^{[35]}$,但模型的系数多为经验参数,不具备实际物理意义,所以应用于不同区域时常需 要进行修正 ${ }^{[30]}$,这导致模拟与试验地点自然人为因素显著不同的区域时精度难以保障.

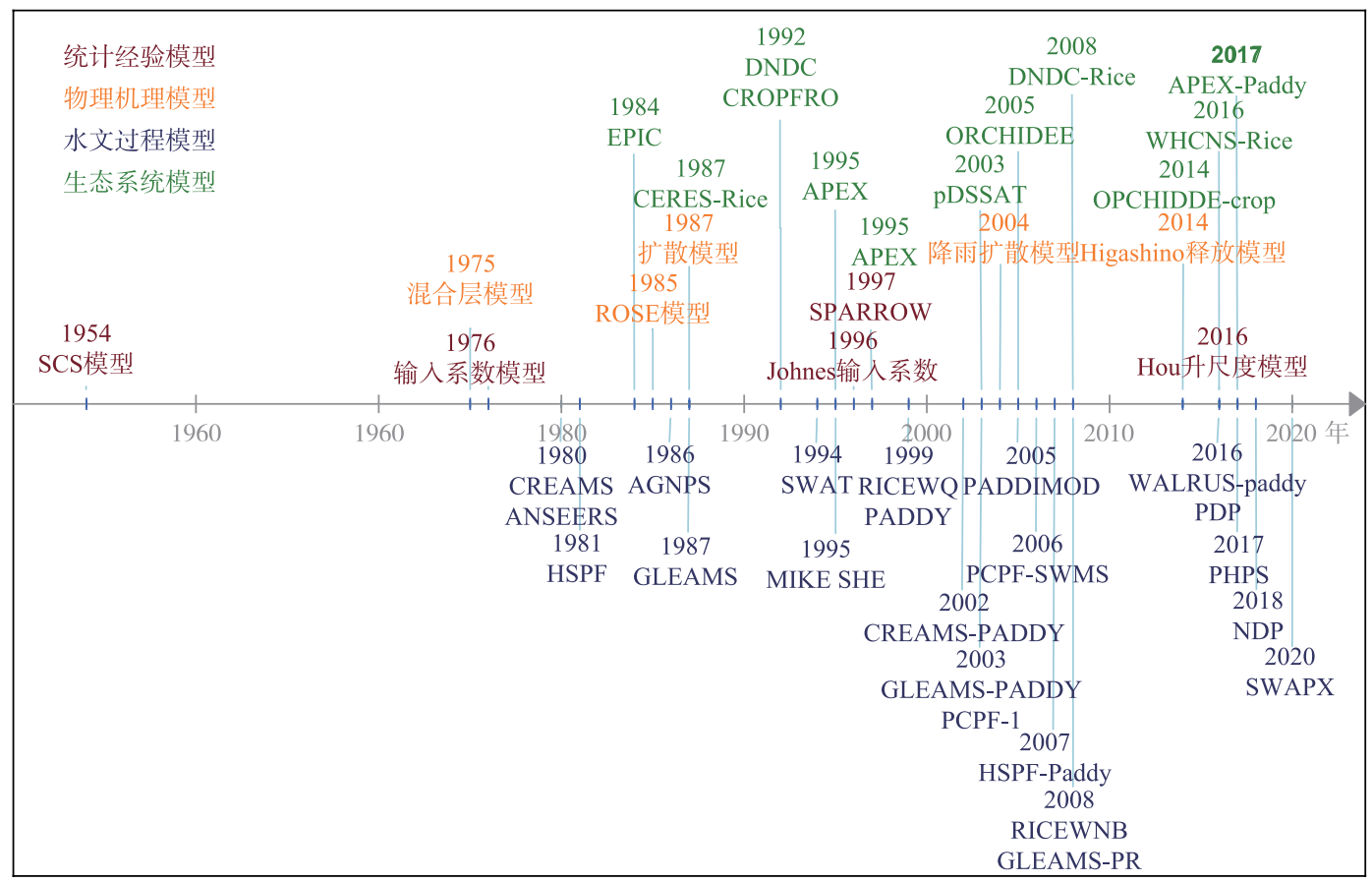

图 2 稻田氮磷径流流失的 4 类模型研发历程

Fig.2 Development progress of 4 types of nitrogen and phosphorus runoff loss models for rice paddies

\section{2 物理机理模型}

物理机理模型比统计经验模型复杂, 考虑了稻田输人、输出和中间迁移转化过程机理, 属于 “白箱” 模 型. 物理机理模型以物质守恒、能量守恒定律为基础, 具有严格理论过程, 模型参数有明确的物理意义, 求解 方法一般是基于设定的初始和边界条件进行数值求解(图 2).

物理机理模型量化了水土界面氮磷迁移转化过程. 这一过程包括了降雨引起的物理侵蚀作用、化学物 质的迁移转化、土壤水一径流混合推移等. 传统的模型主要包括两类, 即混合层模型 ${ }^{[36-37]}$ 和扩散模型 ${ }^{[38-39]}$. 混合层模型假设土壤表层存在一个较薄的混合层, 降水、径流与土壤水在该混合层完全混合, 而扩散模型假 定扩散过程控制土壤和径流之间的化学传递. 这两类模型自提出以来, 关键公式和基本假设不断得到完善, 但往往认为某一种过程起主导性的作用, 且尚未考虑到这些过程的交互影响, 尤其对于降雨的作用考虑不 全. Gao 等 ${ }^{[40-41]}$ 结合了降雨主导的土壤水释放过程和扩散主导的深层土壤迁移到混合层 (交换层) 的过程, 其中, 降雨控制积水一径流层和交换层间的溶质迁移,扩散控制土壤层和交换层间的迁移( 附表 I 和 II ). 理 论上,这些模型可以应用于具有田面积水和田埂的稻田 ${ }^{[42]}$, 但田面水位对降雨溅蚀作用的缓冲作用仍然没 有得到很好的模拟. Higashino 等 $^{[7]}$ 从另一个角度出发, 将雨滴大小、降落速度、雨滴密度 (即单位面积的雨滴 个数) 等参数纳人模型, 细化了稻田水一土界面养分释放过程. 结果表明, 该释放过程对于稻田氮径流流失 的贡献超过 $20 \%$. 尽管物理机理模型的科学假设不断完善, 机理过程逐渐完整, 模拟精度和分辨率逐步提 高, 但输人数据和边界条件要求高, 模拟过程相对复杂 ${ }^{[43]}$,一般是基于少量的室内实验或田间试验进行参数 率定和不确定性分析, 难以在大尺度推广应用.

\section{3 水文过程模型}

水文过程模型在面源污染方面应用很广, 涉及到降雨一径流、土壤侵蚀、污染物迁移转化等过程 (图

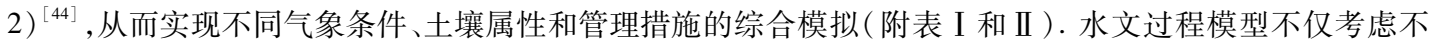


同土壤类型之间的差异,还常对土壤进行分层分区, 以提高模型模拟能力. 从 19 世纪 60 年代开始, 研究者 陆续开发出很多适用于农田和流域尺度的水文过程模型. 农田尺度模型用于单一的土地利用和相对均一的 土壤质地, 是大尺度分布式参数模型建模的基础, 如 CREAMS (chemicals, runoff, and erosion from agricultural management systems) ${ }^{[45]}$ 和 GLEAMS (groundwater loading effects of agricultural management systems) ${ }^{[46]}$; 流域尺 度模型考虑了流域内气候、土地利用、土壤类型、管理措施等的空间异质性, 得到整个流域的污染物输出量, 如 SWAT( soil and water assessment tool) ${ }^{[47]}$ 和 MIKE SHE (MIKE system hydrological European) ${ }^{[48]}$. 这些模型 虽然为过程模型, 但仍然存在很多经验的公式, 如 SWAT 中直接采用了 SCS 和 USLE 模型计算单个响应单元 的径流和土壤侵蚀 (附表 I ). 同时, 这些模型无法准确模拟稻田氮磷径流流失特征, 如 GLEAMS 模型模拟 渗漏时采用的 Storage-Routine 方法、SWAT 将稻田田面水层看作蓄水池的处理等. 鉴于此, 研究者不断对已 有的水文模型进行改进使之具备稻田模拟能力. 例如, RICEWQ (rice water quality) ${ }^{[49]}$ 、PCPF-1 (pesticide concentration in paddy field, v1 $)^{[50]}$ 模型考虑氮磷在田面水中的过程, CREAMS-PADDY ${ }^{[51]}$ 、GLEAMS-PADDY ${ }^{[52]}$ 模型考虑了稻田土壤中的迁移转化行为, GLEAMS-PR (GLEMAS paddy-rice) ${ }^{[53]}$ 和 RICEWNB ( RICE-water and nitrogen balance $)^{[54]}$ 模型还探索了稻田传热和根区土壤氮循环的模拟.

计算机和 $3 \mathrm{~S}$ ( GIS、GPS、RS) 技术的不断发展提供了大量的空间数据和统计数据, 模型的数据处理能力 和应用价值大大提升. 水文过程模型在此背景下从单纯的数值计算程序成为了多功能一体的专业软件系 统, 更多地应用于大尺度稻区的高分辨模拟, 包括氮磷径流流失通量估算和减排措施优化. Yan 等 $^{[55]}$ 在 WALRUS 模型中考虑了稻田的灌溉和排水方式,引人了新的磷模块, 形成了面向圩区的水文过程模型 PHPS (lowland polder hydrology and phosphorus modelling system), 对太湖流域稻区有无抽水情景进行模拟, 并提出 了增加抽水、频繁清淤以及提高稻区范围水体水生植物的覆盖范围等关键减排措施.

水文过程模型具备完善的水文模块和氮磷循环模块,适用于稻区水分和养分循环研究, 探索流域氮磷 流失规律. 这些水文过程模型全面考虑田面水的推流过程以及氮磷在土壤层中的物理化学迁移转化过程, 但多用于日或月尺度模拟, 且常将降雨仅仅视为水量输人而忽略了降雨对土壤的间歇性冲击扰动作用, 使 得稻田氮磷径流流失的模拟结果存在一定偏差. 另外, 水文过程模型对农艺管理措施的参数化较为简单, 对 于大尺度模拟存在较大的不确定性.

\section{4 生态系统模型}

生态系统模型动态描述作物生长及水、氮和磷元素在土壤一作物一大气连续体中的生物地球化学循环 过程 (图 2). 与水文过程模型不同, 其更侧重于水稻的生理生态和生长发育过程, 以及对氮磷输人、气象和 水肥管理的响应. 农田生态系统模型开发起始于 $1960 \mathrm{~s}-1970 \mathrm{~s}^{[56-59]}$, 如美国学者开发了 CERES 模型并整合 到 DSSAT 系统 ${ }^{[60-61]}$. 部分农田生态系统模型针对稻田进行了改进, 如 CERES-Rice ${ }^{[62]}$ 和 WHCNS-Rice ${ }^{[63]}$. 李 长生等 ${ }^{[6-65]}$ 构建了 DNDC 模型 (denitrification-decomposition), 较好地模拟农业生态系统碳一氮一水的生物地 球化学循环. 与这类模型相比, 陆面过程模型引人了农作物模块 ${ }^{[6]}$, 更好体现作物生理生态机制 ${ }^{[67]}$, 例如 ORCHIDEE 模型 (organizing carbon and hydrology in dynamic ecosystems) ${ }^{[68]}$. 王旭辉 ${ }^{[69]}$ 在此基础上开发了 ORCHIDEE-crop 模型以及其参数最优化系统, 实现不同稻区水稻模拟.

生态系统模型具备多时空尺度模拟能力, 如田块尺度的 EPIC 模型 ${ }^{[70]}$ 发展起来的 APEX 模型 (agricultural policy/environmental extender) ${ }^{[71]}$ 可用于小流域尺度, 该模型时空分辨率高, 具有较好的水稻种 植管理措施模块, 已具备响应气候变化和稻田农艺管理措施的模拟能力 ${ }^{[72]}$. 目前, 生态系统模型已经广泛 应用于稻田模拟, 多用于不同情景下水稻生长、水热通量和温室气体的排放模拟, 然而, 氮磷径流流失模拟 能力较弱. Zhao 等 ${ }^{[73]}$ 将 SCS 和 RULSE 引人 DNDC 模型 (附表 I ) ${ }^{[74]}$, 实现稻田氮素径流流失的季节过程模 拟,并应用于上海地区稻田最佳水肥管理措施优化. 相比物理机理模型和水文过程模型, 生态系统模型更关 注养分循环和作物生长发育, 对稻田径流流失的模拟具有浓厚的经验性质, 无法有效响应气候变化和水肥 管理的影响 ${ }^{[75]}$, 难以实现区域普适性 ${ }^{[76]}$. 同时, 由于磷的迁移转化能力相对较弱, 也不参与稻田温室气体的 排放,生态系统模型往往忽略了磷循环过程.

稻田氮磷径流流失发生机制十分复杂, 常常与许多因素 (如气象水文、土地利用、土壤类型、农田管理措 施等) 密切相关. 以上 4 类模型侧重点不同, 模拟氮磷径流流失过程采用的方法和公式表达存在差异, 对于 
驱动数据的要求和适用的范围尺度等也有所区别, 这导致了模拟功能、时空尺度和适应范围方面上的差别. 本文从稻田氮磷径流流失的过程环节出发, 选取 7 个具有代表性的流失模型进行归纳和对比 (附表 I 和 II ). 模型的开发及具体应用可参阅相关研究成果.

\section{5 不确定性评估}

稻田氮磷径流流失具有复杂的发生机制, 受到各类随机因素的影响, 而模型运用相对简单的数学公式 进行表述, 就会造成不确定性. 不确定性主要可以分为随机性、模糊性、灰色性和未确知性 ${ }^{[77]}$. 不确定性研 究旨在理清不确定性来源并试图降低不确定性, 提高模型预测能力. 不确定性分析方法和理念随着稻田流 失模型的改进也在不断发展. 但模型的不确定性十分复杂且相互关联, 目前的研究仍偏向单一过程和案例 的不确定性, 缺乏推广能力 ${ }^{[78]}$. 目前, 稻田氮磷径流流失模型的不确定性研究包括模型和数据两个方面 ${ }^{[79]}$.

首先, 模型不确定性包括模型结构和模型参数的不确定性. 对于稻田流失机理和过程认识的不完整, 数 学表达中的简化处理, 模拟的尺度问题, 全局最优的参数估值过程中伴随的参数敏感性、相关性、阈值等因 素的影响, 都会造成这种不确定性 ${ }^{[20]}$. 在模型改进历程中, 流失机理逐渐完善, 考虑过程逐渐周全, 模型也 具备了模拟更大时空尺度的能力. 如 Gao 等 ${ }^{[00-41]}$ 在物理模型中考虑降雨引起的释放作用后, 研究者 ${ }^{[42-43,80-81]}$ 又陆续考虑了渗透、化学转化等过程和不同土壤、水力条件下的适用性, 降低了模型结构的不确定性. “试错 法” 是率定稻田氮磷径流流失模型参数最传统的方法, 该方法费时费力, 且带有很大的主观性和偶然性 ${ }^{[47]}$. 随着计算机技术的发展, 贝叶斯法 ${ }^{[82]} 、 \mathrm{SCE}^{-U} \mathrm{UA}^{[83]} 、 \mathrm{SCPSO}^{[84]}$ 等自动优选方法逐渐得到开发和应用, 对于模 型参数的不确定性研究具有重要意义.

其次, 数据不确定性包括输人数据和验证数据 (即输出数据) 的不确定性. 稻田径流流失模型的输人数 据常包括基础地理、降雨灌溉、土地利用和土壤属性、农艺管理措施等, 而模型还需要径流通量、氮磷浓度等 观测数据进行校准和验证. 受制于数据的时空尺度和分辨率, 模拟结果存在不确定性. 对于基础地理、降雨 等数据, 目前常采用改变其输人的空间分布和尺度的方法, 考察其对于最终结果的影响 ${ }^{[20]}$, 而氮磷浓度、径 流等观测数据的测量误差受实验方法和监测手段的制约, 仍没有较好的不确定性分析方法. 有研究者尝试 采用随机变量 ${ }^{[85]}$ 或加人随机干扰数据 ${ }^{[86]}$ 来量化数据的不确定性.

\section{3 模型关键问题}

\section{1 发生机制的参数化}

上述 4 类模型的稻田氮磷径流流失发生机制还需要更好的参数化. 目前对于溶质从土壤迁移到地表径 流的机理及这些过程对于自然人文相关因素的定量响应的认识仍然有限. 比如不同土壤类型、溶质、土壤渗 透率、地表覆被等因素下降雨径流混合层的深度都会存在差异 ${ }^{[87-88]}$, 目前仍然没有有效的方法进行精确定 量 ${ }^{[89]}$. 土壤一水释放迁移速率、传质系数等的区域适用性仍需要研究证实. 当未来气候超过当前波动范围 时,基于现在实验条件下得到的稻田氮磷径流流失响应规律是否有效也是关键问题 ${ }^{[00]}$.

除此之外, 大量的物理机理模型往往针对坡地和旱地, 导致对于具有特殊性的稻田 (较高的田面水位、 淹水环境下特殊的土壤分层等) 的模拟效果较差, 而水文过程和生态系统模型对部分复杂过程难以进行微 观定量模拟, 这意味着稻田氮磷流失的发生机制以及参数化的研究仍然存在诸多空白. 虽然少量研究开始 关注降雨引起水土界面氮磷释放的关键作用, 但释放的间歇性、孔隙水浓度、田面水中推流输移时再吸附和 再沉淀等过程仍然缺乏有效参数化, 无法区分常规降水和极端降水变化 (频率、强度、持续时间等增加 ${ }^{[91]}$ ) 的影响.

稻田氮磷径流流失是氮磷循环过程的之一, 并非孤立, 而与氮磷利用、氮磷损失、水一土一气界面过程等 密切相关. 例如, 作物生理生态过程影响蒸散发和氮磷吸收利用, 从而改变田面水位和田面水氮磷浓度等径 流流失的初始条件 ${ }^{[7]}$. 然而, 经验、物理机理和水文过程模型缺乏作物生理生态过程模拟能力, 而农田生态 系统模型缺乏稻田氮磷径流流失对作物生理生态的响应模拟能力. 因此, 不同类型模型的耦合可能是解决 这类问题的方法之一.

\section{2 模型参数的合理化}

模型率定和验证是保证参数合理的关键步骤 ${ }^{[92]}$. 参数的选择往往被限制在预定义的范围内, 再根据观 
测数据进行最优值的选定, 以反映参数的区域适应性 ${ }^{[93]}$. 传统的生态系统模型, 通常在站点尺度上校准, 升 尺度到大尺度 ${ }^{[94]}$, 因此, 需要高分辨率、多年多站点观测数据进行模型率定和验证, 降低模型预测的不确定 性. 对于水文过程模型, 需要选择具有代表性的观测站点数据来反映空间分异性, 但常规的观测数据难以满 足要求, 而人工采样难以提高时间和空间分辨率. 此外, 目前率定数据多采用实际环境状态变量的观测值, 即“硬约束” 观测数据. 最近研究学者提出 “软约束” 观测数据 ${ }^{[95-96]}$, 即与模拟的各独立过程和平衡有关的信 息 ${ }^{[97]}$,这将是提高模型参数的合理化的可行途径.

稻田氮磷径流流失模型普遍缺乏针对每个发生过程 (释放、径流、输移) 的逐一率定, 且氮磷循环过程的 通量也难以同步率定, 这就造成为了模拟氮磷径流流失过程时牺牲其他过程的模拟精度, 从而降低模型整 体的可信度. 对于高度非线性模型, 很可能只在参数空间搜寻到一组最佳组合 ${ }^{[69]}$. 目前, 对于流失模型多采 用单目标函数进行参数率定, 基于有限特征指标的多目标全局优化方法尚未能考虑目标组合优化和多目标 函数对模型参数集的影响,更难以解决异参同效问题 ${ }^{[93]}$.

目前, 参数自动优选方法得到了普遍应用 ${ }^{[82-83]}$, 但基于启发式搜索的模型参数自动优选算法仍存在诸 多问题, 搜索算法的盲目随机游动性问题突出, 搜索效率较低, 容易陷人局部陷荫 ${ }^{[98]}$. 王旭辉 ${ }^{[69]}$ 利用粒子滤 波算法构建了 ORCHIDEE-crop 模型的参数最优化系统, 使得模型具有鲁棒性, 但由于观测数据误差获取的 限制, 仍无法很好做到上述的多变量同步优化, 且大规模计算能力也存在技术瓶颈. 目前针对稻田的模型多 是采用成熟的参数率定方法, 考虑到稻田的特殊性, 未来仍需要采用更有效的方法来分解模型参数、模型结 构、输人数据集、误差结构和概率对模型模拟结果的不确定性. 从田块尺度到流域、区域、全球尺度, 主控因 素也会发生变化, 参数值可能不适用, 需要进行建立传递函数实现参数升尺度 ${ }^{[21]}$.

\section{3 农艺管理与措施的模块化}

农艺管理措施显著改变稻田氮磷径流流失过程 ${ }^{[99]}$, 但相关模块开发和改进仍然存在极大的空间. 稻田 的灌溉、排水方式与旱地显著不同, 并直接影响着稻田氮磷径流流失. Yang 等 ${ }^{[100]}$ 发现优化灌溉相比于传统 的大水漫灌能够显著减少稻田 $24 \%$ 的氮流失, 而移栽前田间被动排水也是一大贡献因素 ${ }^{[101]}$. 然而, 目前的 模型缺乏自动灌排制度的模拟能力. 最近研制了针对稻田的灌溉排水控制方案模拟 ${ }^{[101-102]}$, 但过于简单, 无 法涵盖灌排制度的多样性和区域性. 这类模拟还受到实验观测的限制, 未能进行同步率定和长期的验证, 仍 无法直接外推到实验区以外的稻田. 此外, 目前的模型中还缺乏节水灌溉和田一沟一塘的联合调控的措施模 拟. 例如, 排水沟渠和塘堰系统是稻田与水体之间的一个过渡带, 能够在减少稻田进人下游水体的氮磷流失 方面起到重要作用 ${ }^{[103]}$. 稻区沟、塘数量多且形状极其不规则, 田一沟一塘的联合运行多种多样, 如何识别其 关键参数及其定量化是解决不同措施下稻田氮磷径流流失模拟问题的核心 ${ }^{[102]}$.

稻田的总氮流失量还受种植制度、施肥制度 (量、类型、时间和方式) 的影响 ${ }^{[104]}$. 例如, 深施相比表施能 显著减少稻田氮径流流失的 $48 \% \sim 72 \%$. 不管是统计经验模型还是过程模型 ${ }^{[5,25,69]}$, 模型缺乏稻田氮磷径流 流失对移栽和收获日期、施肥制度 (量、类型、时间和方式)、秸秆还田比例等管理措施的响应能力. 另外, 对 于站点或小尺度而言, 农艺管理措施可以通过调查直接输人, 但对于大尺度而言则难以获取到调查数据. 如 何建立种植制度 (轮作与否、移栽和收获时间)、施肥管理 (量、类型、时间、位置), 耕作 (耕作、少耕、免耕) 等 大尺度高分辨数据集也是农艺管理与措施模块化的另一个关键.

\section{4 展望}

自 1950s 以来, 稻田氮磷径流流失模型研究已经取得了显著的成果, 在全球范围得以广泛应用, 为稻田 流失机理解析、通量估算及减排措施做出了重大贡献. 然而, 稻田氮磷径流流失模型在发生机制、模型参数 和农艺管理措施等关键问题方面尚存在不足, 仍有较多的改进空间, 本文总结前述, 展望未来改进与发展的 趋势如下:

1) 提高观测数据质量和过程机理研究. 稻田氮磷径流流失过程机理复杂, 模拟参数众多, 不确定性大. 未来将需要改进实验设计和监测技术, 获得更高时间分辨率、全通量、多站点、多种自然人文环境驱动、以及 不同水稻品种和农艺管理措施下的实验观测数据, 并通过机制平台共享数据, 以达到模型应用的标准化和 模型不确定性的降低. 另外, 增强人工模拟试验与模型模拟的结合, 进一步加强稻田氮磷流失形成过程各个 
环节的机理研究, 对稻田释放、吸附解析过程和混合层深度等进行研究量化. 关注农艺管理措施的发展和改 进 ${ }^{[13,69]}$, 实现农艺措施的模块化和参数化.

2) 加强模型的开发改进与先进技术的集成. 计算机技术的发展和 $3 \mathrm{~S}$ 的应用给稻田氮磷流失模型的开 发思路和手段带来了技术创新和革命. 近年来, 人工智能和机器学习取得了飞速的发展, 目前已经存在有机 结合过程模型和智能模型的相关研究 ${ }^{[105]}$, 但尚未得到广泛的应用. 基于 CPU 和 GPU 异构并行的高性能计 算技术也取得了显著的发展 ${ }^{[106]}$, 能够显著提高模型计算性能,增强模型可视化, 推动稻田氮磷流失模型与 先进技术手段的集成和融合.

3) 加强氮磷流失不同过程、不同模块、不同模型类型之间的耦合. 稻田的氮磷流失是不同因素综合作用 的结果, 包含了复杂的物理化学生物过程的耦合和互馈, 一些过程是相同的, 在模型中互相借鉴, 如植物生 长过程;一些过程是相关的, 如水文过程与生态过程, 因此, 不同学科的模型耦合成为必然. 目前,生态模型 对于稻田氮磷迁移转化流失模拟仍较为简单, 而水文模型用于稻田模拟时对于作物生长和管理措施考虑还 不够全面. 最近就有研究者将生态模型 APEX 和水文模型 SWAT 模型结合起来, 开发了混合模型 SWAPX, 来探索气候变化情景下稻区最优管理措施 ${ }^{[107]}$. 因此,耦合稻田生态系统模块和流域水文模型, 实现水文和 稻田氮磷循环系统模拟, 建立多模型集合框架 ${ }^{[108]}$,有利于提高多种胁迫压力 (包括极端气候事件)下模型模 拟精度 ${ }^{[109]}$.

\section{5 附录}

附表 I 和 II 见电子版( DOI : 10.18307/2021.0202).

\section{6 参考文献}

[ 1 ] Zhao C, Piao S, Wang X et al. Plausible rice yield losses under future climate warming. Nature Plants, 2017, 3(1). DOI: $10.1038 /$ nplants.2016.202.

[ 2 ] FAO. 2018. FAOSTAT Crops data. http://www.fao.org/faostat/en/\#data/QC.

[ 3 ] IFA, IPNI. Assessment of fertilizer use by crop at the global level 2014-2014/15. Paris, France: IFA, 2017.

[ 4 ] Chen F, Hou L, Liu M et al. Net anthropogenic nitrogen inputs (NANI) into the Yangtze River basin and the relationship with riverine nitrogen export. Journal of Geophysical Research-Biogeosciences, 2016, 121 ( 2) : 451-465. DOI: 10. 1002/2015jg003186.

[ 5 ] Hou X, Zhan X, Zhou F et al. Detection and attribution of nitrogen runoff trend in China's croplands. Environmental Pollution, 2018, 234: 270-278. DOI: 10.1016/j.envpol.2017.11.052.

[ 6 ] Sinha E, Michalak AM, Calvin KV et al. Societal decisions about climate mitigation will have dramatic impacts on eutrophication in the 21st century. Nature Communications, 2019, 939(10). DOI: 10.1038/s41467-019-08884-w.

[ 7 ] Higashino M, Stefan HG. Modeling the effect of rainfall intensity on soil-water nutrient exchange in flooded rice paddies and implications for nitrate fertilizer runoff to the Oita River in Japan. Water Resources Research, 2014, 50(11) : 86118624. DOI: $10.1002 / 2013 \mathrm{wr} 014643$.

[ 8 ] Fu CD, Liang XQ, Zhao Y et al. Temporal changes of P concentrations in floodwater under different soil types and P fertilizer rates. Journal of Soil and Water Conservation, 2014, 28(4): 7-12. [傅朝栋, 梁新强, 赵越等. 不同土壤类型及施 磷水平的水稻田面水磷素浓度变化规律. 水土保持学报, 2014, 28(4) : 7-12.]

[ 9 ] Yang Y, Zhang M, Zheng L et al. Controlled-release urea for rice production and its environmental implications. Journal of Plant Nutrition, 2013, 36(5) : 781-794. DOI: 10.1080/01904167.2012.756892.

[10] Liang XQ, Chen YX, Nie ZY et al. Mitigation of nutrient losses via surface runoff from rice cropping systems with alternate wetting and drying irrigation and site-specific nutrient management practices. Environmental Science and Pollution Research, 2013, 20(10) : 6980-6991. DOI: 10.1007/s11356-012-1391-1.

[11] Zhuang Y, Zhang L, Li S et al. Effects and potential of water-saving irrigation for rice production in China. Agricultural Water Management, 2019, 217: 374-382. DOI: 10.1016/j.agwat.2019.03.010.

[12] He WY, Mao M. Research and application progresses on rice water quality (RICEWQ) model. Chinese Journal of Applied Ecology , 2019, 30(11) : 3963-3970. DOI: 10.13287/j.1001-9332.201911.016. [贺文艳, 毛萌. 稻田水质模型研究和 
应用进展. 应用生态学报, 2019, 30(11) : 3963-3970.]

[13] Zhang XX, Zhou S, Sun HF et al. Advance and application of DNDC model in paddy ecosystem. Acta Agriculturae Shang$h a i, 2019,35$ (1) : 114-122. [ 张鲜鲜, 周胜, 孙会峰等. DNDC 模型在稻田生态系统中的研究进展及应用. 上海农 业学报, 2019, 35(1): 114-122.]

[14] Li CL, Hu YM, Liu M et al. Urban non-point source pollution: Research progress. Chinese Journal of Ecology, 2013,32 (2)：492-500. [李春林, 胡远满, 刘沝等. 城市非点源污染研究进展. 生态学杂志, 2013, 32(2) : 492-500.]

[15] Li YJ, Song JB, Li JK et al. Research and application progress of four typical non-point source pollution models. Water Resources and Power, 2019, 37(3) : 21-24, 32. [李亚娇, 宋佳宝, 李家科等. 四种典型非点源污染模型研究与应用进 展. 水电能源科学, 2019, 37(3): 21-24, 32.]

[16] Rosenzweig C, Elliott J, Deryng D et al. Assessing agricultural risks of climate change in the 21 st century in a global gridded crop model intercomparison. Proceedings of the National Academy of Sciences of the United States of America, 2014, 111(9) : 3268-3273. DOI: 10.1073/pnas.1222463110.

[17] Shi X, Wu L, Chen W et al. Solute transfer from the soil surface to overland flow a review. Soil Science Society of America Journal, 2011, 75(4) : 1214-1225. DOI: 10.2136/sssaj2010.0433.

[18] Wu JS, Ge TD, Hu YJ. A review on the coupling of bio-geochemical process for key elements and microbial regulation mechanisms in paddy rice ecosystems. Acta Ecologica Sinica, 2015, 35(20): 6626-6634. [ 吴金水, 葛体达, 胡亚军. 稻田土壤关键元素的生物地球化学耦合过程及其微生物调控机制. 生态学报, 2015, 35(20): 6626-6634.]

[19] Kuypers MMM, Marchant HK, Kartal B. The microbial nitrogen-cycling network. Nature Reviews Microbiology, 2018,16 (5) : 263-276. DOI: $10.1038 /$ nrmicro.2018.9.

[20] Liao Q, Shen ZY. Uncertainties in agricultural non-point source pollution simulation: Research progress. Chinese Journal of Ecology, 2011, 30(7) : 1542-1550. [廖谦, 沈珍瑶. 农业非点源污染模拟不确定性研究进展. 生态学杂志, 2011, 30(7): $1542-1550$.

[21] Lai XJ. A review of integrated water quality modeling for a watershed. Progress in Geography, 2019, 38(8) : 1123-1135. [赖锡军. 流域水环境过程综合模拟研究进展. 地理科学进展, 2019, 38(8): 1123-1135.]

[22] Shi XR, Liang H, Zhou F et al. Sensitivity analysis and parameter estimation for soil-rice system Model. Transactions of the Chinese Society for Agricultural Machinery, 2020, (1): 1-16. [史崟苾, 梁浩, 周丰等. 稻田土壤-作物系统模型参数 敏感性分析与模型验证. 农业机械学报, 2020, (1): 1-16.]

[23] Li CS ed. Biogeochemistry: Scientific fundamentals and modeling approach. Beijing: Tsinghua University Press, 2016. [李长生. 生物地球化学:科学基础与模型方法. 北京: 清华大学出版社, 2016.]

[24] Brar HS, Bhullar MS. Nutrient uptake by direct seeded rice and associated weeds as influenced by sowing date, variety and weed control. Indian Journal of Agricultural Research, 2013, 47 (4) : 353-358.

[25] Hou X, Zhou F, Leip A et al. Spatial patterns of nitrogen runoff from Chinese paddy fields. Agriculture, Ecosystems and Environment, 2016, 231: 246-254. DOI: 10.1016/j.agee.2016.07.001.

[26] Yang R, Tong JX, Wei WS et al. Experimental study on nitrogen and phosphorous loss in soil. Journal of Irrigation and Drainage, 2016, 35(8) : 9-15. [杨瑞, 童菊秀, 魏文硕等. 土壤中氮磷流失试验研究. 灌溉排水学报, 2016, 35 (8) : 9-15.]

[27] Xu Y, Su B, Wang H et al. Analysis of the water balance and the nitrogen and phosphorus runoff pollution of a paddy field in situ in the Taihu Lake basin. Paddy and Water Environment, 2020, 18(2) : 385-398. DOI: 10.1007/s10333-02000789-5.

[28] Xia L, Lam SK, Wang S et al. Optimizing nitrogen fertilization rate to enhance soil carbon storage and decrease nitrogen pollution in paddy ecosystems with simultaneous straw incorporation. Agriculture Ecosystems \& Environment, 2020, 298 : 106968. DOI: $10.1016 /$ j.agee.2020.106968.

[29] SCS ed. National Engineering Handbook, Section 4: Hydrology, soil conservation service. Washington, DC: US Department of Agriculture, 1956.

[30] Tian P, Chen YX, Tian GM et al. Estimation of N loss loading by runoff from paddy field during submersed period in Hangjiahu area. Chinese Journal of Applied Ecology, 2006, 17(10): 1911-1917. [田平, 陈英旭, 田光明等. 杭嘉湖地 区淹水稻田氮素径流流失负荷估算. 应用生态学报, 2006, 17(10): 1911-1917.]

[31] Choi D, Park H, Kim YJ et al. Curve numbers for rice paddies with different water management practices in Korea. Jour- 
nal of Irrigation \& Drainage Engineering, 2019, 145(5) : 06019003. DOI: 10.1061/( ASCE) IR.1943-4774.0001382.

[32] Lian H, Yen H, Huang JC et al. CN-China: Revised runoff curve number by using rainfall-runoff events data in China. Water Research, 2020, 177. DOI: 10.1016/j.watres.2020.115767.

[33] Johnes PJ. Evaluation and management of the impact of land use change on the nitrogen and phosphorus load delivered to surface waters: The export coefficient modelling approach. Journal of Hydrology, 1996, 183(3/4) : 323-349. DOI: 10. 1016/0022-1694( 95 )02951-6.

[34] Smith RA, Schwarz GE, Alexander RB. Regional interpretation of water-quality monitoring data. Water Resources Research, 1997, 33(12) : 2781-2798. DOI: 10.1029/97WR02171

[35] Sun X, Liang X, Zhang F et al. A GIS-based upscaling estimation of nutrient runoff losses from rice paddy fields to a regional level. Journal of Environmental Quality, 2016, 45(6) : 1865-1873. DOI: 10.2134/jeq2016.05.0181

[36] Bruce RR, Harper LA, Leonard RA et al. A model for runoff of pesticides from upland watersheds. Journal of Environmental Quality, 1975, 4(4) : 541-548. DOI: 10.2134/jeq1975.00472425000400040024x.

[37] Ahuja LR, Lehman OR. The extent and nature of rainfall-soil interaction in the release of soluble chemicals to runoff. Journal of Environmental Quality, 1983, 12: 34-40. DOI: 10.2134/jeq1983.00472425001200010005x.

[38 ] Parr AD, Richardson C, Lane DD et al. Pore water uptake by agricultural runoff. Journal of Environmental EngineeringAsce, 1987, 113(1) : 49-63. DOI: 10.1061/(asce) 0733-9372(1987)113:1(49).

[39] Wallach R, Grigorin G, Rivlin J. A comprehensive mathematical model for transport of soil-dissolved chemicals by overland flow. Journal of Hydrology, 2001, 247 (1/2) : 85-99. DOI: 10.1016/s0022-1694( 01) 00365-1.

[40] Gao B, Todd WM, Steenhuis TS et al. Rainfall induced chemical transport from soil to runoff: theory and experiments. Journal of Hydrology, 2004, 295(1/2/3/4) : 291-304. DOI: 10.1016/j.jhydrol.2004.03.026.

[41] Gao B, Walter MT, Steenhuis TS et al. Investigating raindrop effects on transport of sediment and non-sorbed chemicals from soil to surface runoff. Journal of Hydrology, 2005, 308(1/2/3/4) : 313-320. DOI: 10.1016/j.jhydrol.2004.11.007.

[42] Walter MT, Gao B, Parlange JY. Modeling soil solute release into runoff with infiltration. Journal of Hydrology, 2007, 347 (3/4) : 430-437. DOI: 10.1016/j.jhydrol.2007.09.033.

[43] Li J, Tong J, Xia C et al. Numerical simulation and experimental study on farmland nitrogen loss to surface runoff in a raindrop driven process. Journal of Hydrology, 2017, 549 : 754-768. DOI: 10.1016/j.jhydrol.2017.01.035.

[44] USEPA. National management measures to control nonpoint source pollution from agriculture. Washington, DC: US Environmental Protection Agency, Office of Water, 2003.

[45] Knisel WG. CREAMS: A field scale model for chemicals, runoff, and erosions from agricultural management systems. Washington DC: Conservation Research Report No.26, USDA, 1980.

[46] Leonard RA, Knisel WG, Still DA. GLEAMS: Groundwater loading effects of agricultural management systems. Transactions of the Asabe, 1987, 30(5): 1403-1418. DOI: 10.13031/2013.30578.

[47] Gassman PW, Reyes MR, Green CH et al. The soil and water assessment tool: Historical development, applications, and future research directions. Transactions of the Asabe, 2007, 50(4) : 1211-1250. DOI: 10.13031/2013.23637.

[48] Refsgaard JC, Storm B. MIKE SHE//Singh VP, Frevert DK eds. Computer models of watershed hydrology. Highlands Ranch, Colorado: Water Resources Publications, 1995.

[49] Williams WM, Ritter AM, Cheplick JM et al. RICEWQ: Pesticide runoff model for rice crops, users manual and program documentation Version 1.6.1. S.E.Leesburg, VA Waterborne Environmental, Inc, 1999.

[50] Watanabe H, Takagi K, Vu SH. Simulation of mefenacet concentrations in paddy fields by an improved PCPF-1 model. Pest Management Science, 2006, 62(1) : 20-29. DOI : 10.1002/ps.1115.

[51] Chin YM, Park SW, Kim SM et al. Nutrient loads estimation at paddy field using CREAMS-PADDY model. Journal of Korean Society of Rural Planning, 2002, 8(1): 60-68.

[52] Chung SO, Kim HS, Kim JS. Model development for nutrient loading from paddy rice fields. Agricultural Water Management, 2003, 62(1) : 1-17. DOI: 10.1016/s0378-3774(03)00078-7.

[53] Aschonitis VG, Antonopoulos VZ. Evaluation of the water balance and the soil and ponding water temperature in paddy-rice fields with the modified GLEAMS model. Crete: Proceedings of Agricultural Engineering Conference, 2008: 15.

[54] Antonopoulos VZ. Modelling of water and nitrogen balances in the ponded water and soil profile of rice fields in Northern Greece. Agricultural Water Management, 2010, 98(2) : 321-330. DOI: 10.1016/j.agwat.2010.08.026. 
[55] Yan R, Li L, Gao J. Modelling the regulation effects of lowland polder with pumping station on hydrological processes and phosphorus loads. Science of the Total Environment, 2018, 637: 200-207. DOI: 10.1016/j.scitotenv.2018.04.389.

[56] De Wit CT. Photo synthesis of leaf canopies. Agricultural Research Report, 1965, 663: 1-56.

[57] Duncan WG, Loomis RS, Williams WA et al. A model for simulating photosynthesis in plant communities. Hilgardia, 1967, 38(4) : 181-205. DOI: 10.3733/hilg.v38n04p181.

[58] Bouman BAM, van Laar HH. Description and evaluation of the rice growth model ORYZA2000 under nitrogen-limited conditions. Agricultural Systems, 2006, 87(3) : 249-273. DOI: 10.1016/j.agsy.2004.09.011.

[59] Asseng S, Keating BA, Fillery IRP et al. Performance of the APSIM-wheat model in Western Australia. Field Crops Research, 1998, 57(2) : 163-179. DOI: 10.1016/s0378-4290(97)00117-2.

[60] Richter GM, Semenov MA. Modelling impacts of climate change on wheat yields in England and Wales: assessing drought risks. Agricultural Systems, 2005, 84(1) : 77-97. DOI: 10.1016/j.agsy.2004.06.011.

[61] Jones JW, Hoogenboom G, Porter CH et al. The DSSAT cropping system model. European Journal of Agronomy, 2003,18 (3/4) : 235-265. DOI: 10.1016/s1161-0301(02)00107-7.

[62] Mahmood R. Air temperature variations and rice productivity in Bangladesh: a comparative study of the performance of the YIELD and the CERES-Rice models. Ecological Modelling, 1998, 106(2/3) : 201-212. DOI: 10.1016/s0304-3800(97) 00192-0.

[63] Liang H, Hu K, Batchelor WD et al. An integrated soil-crop system model for water and nitrogen management in North China. Scientific Reports, 2016, 6(25755). DOI: 10.1038/srep25755.

[64] Li CS, Frolking S, Frolking TA. A model of nitrous oxide evolution from soil driven by rainfall events. 1. Model structure and sentivity. Journal of Geophysical Research-Atmospheres, 1992, 97( D9) : 9759-9776. DOI: 10.1029/92jd00509.

[65] Li CS, Frolking S, Frolking TA. A model of nitrous oxide evolution from soil driven by rainfall events. 2. Model applications Journal of Geophysical Research-Atmospheres, 1992, 97( D9) : 9777-9783. DOI: 10.1029/92jd00510.

[66] Drewniak B, Song J, Prell J et al. Modeling agriculture in the Community Land Model. Geoscientific Model Development, 2013, 6: 495-515. DOI: 10.5194/gmd-6-495-2013.

[67] Piao S, Ciais P, Friedlingstein P et al. Spatiotemporal patterns of terrestrial carbon cycle during the 20th century. Global Biogeochemical Cycles, 2009, 23(GB4026). DOI: 10.1029/2008gb003339.

[68] Krinner G, Viovy N, de Noblet-Ducoudre N et al. A dynamic global vegetation model for studies of the coupled atmosphere-biosphere system. Global Biogeochemical Cycles, 2005, 19(1). DOI: 10.1029/2003gb002199.

[69] Wang XH. Impacts of environmental change on rice ecosystems in China: development, optimization and application of ORCHIDEE-crop model[Dissertation]. Beijing: Peking University, 2015. [王旭辉. 中国水稻生态系统对环境变化的 响应—OORCHIDEE-crop 模型的开发、校准和应用 [学位论文]. 北京: 北京大学, 2015.]

[70] Williams JR, Renard KG, Dyke PT. EPIC-A new method for assessing erosions effect on soil productivity. Journal of Soil and Water Conservation, 1983, 38(5) : 381-383. DOI: 10.1016/0016-7061(83)90021-6.

[71] Gassman PW, Williams JR, Benson VW et al. Historical development and applications of the EPIC and APEX Models. Ottawa: 2004 ASAE/CSAE annual international meeting, 2005. DOI: 10.13031/2013.17074.

[72] Kamruzzaman M, Hwang S, Choi SK et al. Evaluating the impact of climate change on paddy water balance using APEXPaddy Model. Water, 2020, 12(3) : 852. DOI: 10.3390/w12030852.

[73] Zhao Z, Zhang H, Li C et al. Quantifying nitrogen loading from a paddy field in Shanghai, China with modified DNDC model. Agriculture Ecosystems \& Environment, 2014, 197: 212-221. DOI: 10.1016/j.agee.2014.08.002.

[74] Deng J, Zhu B, Zhou Z et al. Modeling nitrogen loadings from agricultural soils in southwest China with modified DNDC. Journal of Geophysical Research-Biogeosciences, 2011, 116(G02020). DOI: 10.1029/2010jg001609.

[75] Soussana JF, Graux AI, Tubiello FN. Improving the use of modelling for projections of climate change impacts on crops and pastures. Journal of Experimental Botany, 2010, 61(8) : 2217-2228. DOI: 10.1093/jxb/erq100.

[76] Martre P, Wallach D, Asseng S et al. Multimodel ensembles of wheat growth: many models are better than one. Global Change Biology, 2015, 21(2) : 911-925. DOI: 10.1111/gcb.12768.

[77] Li N, Mclaughlin D, Kinzelbach W et al. Using an ensemble smoother to evaluate parameter uncertainty of an integrated hydrological model of Yanqi basin. Journal of Hydrology, 2015, 529: 146-158. DOI: 10.1016/j.jhydrol.2015.07.024.

[78 ] Li T, Hasegawa T, Yin X et al. Uncertainties in predicting rice yield by current crop models under a wide range of climatic 
conditions. Global Change Biology, 2015, 21(3) : 1328-1341. DOI: 10.1111/gcb.12758.

[79] EPA. Guidance on the development, evaluation, and application of environmental models. EPA/100/K- 09/003. US Environmental Protection Agency, 2009.

[80] Walker JD, Walter MT, Parlange JY et al. Reduced raindrop-impact driven soil erosion by infiltration. Journal of Hydrolo$g y, 2007,342(3 / 4)$ : 331-335. DOI: 10.1016/j.jhydrol.2007.06.003.

[81] Tong J, Yang J, Hu BX et al. Experimental study on soluble chemical transfer to surface runoff from soil. Environmental Science and Pollution Research, 2016, 23(20) : 20378-20387. DOI: 10.1007/s11356-016-7248-2.

[82] Jin X, Xu CY, Zhang Q et al. Parameter and modeling uncertainty simulated by GLUE and a formal Bayesian method for a conceptual hydrological model. Journal of Hydrology, 2010, 383(3/4) : 147-155. DOI : 10.1016/j.jhydrol.2009.12.028.

[83] Duan QY, Sorooshian S, Gupta V. Effective and efficient global optimization for conceptual rainfall-runoff models. Water Resources Research, 1992, 28(4) : 1015-1031. DOI: 10.1029/91wr02985.

[84] Wang SG ed. Studies on parameter estimation methods for hydrological model and associated uncertainties. Zhengzhou: Yellow River Water Conservancy Press, 2010. [王书功. 水文模型参数估计方法及参数估计不确定性研究. 郑州: 黄 河水利出版社, 2010.]

[85] Kavetski D, Kuczera G, Franks SW. Calibration of conceptual hydrological models revisited: 1. Overcoming numerical artefacts. Journal of Hydrology, 2006, 320(1/2) : 173-186. DOI: 10.1016/j.jhydrol.2005.07.012.

[86] Chiang S, Tachikawa Y, Takara K. Hydrological model performance comparison through uncertainty recognition and quantification. Hydrological Processes, 2007, 21(9): 1179-1195. DOI: 10.1002/hyp.6678.

[87] Yang T, Wang Q, Xu D et al. A method for estimating the interaction depth of surface soil with simulated rain. Catena, 2015, 124: 109-118. DOI: 10.1016/j.catena.2014.09.009.

[88] Yang T, Wang Q, Liu Y et al. A comparison of mathematical models for chemical transfer from soil to surface runoff with impact of rain. Catena, 2016, 137: 191-202. DOI: 10.1016/j.catena.2015.09.014.

[89] Zhang Y, Zhang X, Shi S, et al. Determining the depth of mixing layer in which soil solute releasing from soil to surface runoff on the unsaturated loess slope under artificial rainfall condition. Journal of Soils and Sediments, 2019, 20(1) : 153165. DOI: $10.1007 / \mathrm{s} 11368-019-02370-y$.

[90] Mora C, Frazier AG, Longman RJ et al. The projected timing of climate departure from recent variability. Nature, 2013, 502(7470) : 183-187. DOI: 10.1038/nature12540.

[91] Barros V, Stocker TF. Managing the risks of extreme events and disasters to advance climate change adapation: Special report of the Intergovernmental Panel on Climate Change. Journal of Endocrinology \& Metabolism, 2012, 18(6) : 586-599.

[92] ASABE. Guidelines for calibrating, validating, and evaluating hydrologic and water quality ( H/WQ) models. The American Society of Agricultural and Biological Engineers. ASABE EP621, 2017.

[93] Adeyeri OE, Laux P, Arnault J et al. Conceptual hydrological model calibration using multi-objective optimization techniques over the transboundary Komadugu-Yobe basin, Lake Chad Area, West Africa. Journal of Hydrology-Regional Studies, 2020, 27 : 100655. DOI: 10.1016/j.ejrh.2019.100655.

[94] Asseng S, Ewert F, Martre P et al. Rising temperatures reduce global wheat production. Nature Climate Change, 2015,5 (2) : 143-147.

[95] Seibert J, McDonnell JJ. The quest for an improved dialog between modeler and experimentalist. Water Science and Applications, 2003, 6: 301-316. DOI: 10.1002/9781118665671.ch22.

[96] Arnold JG, Youssef MA, Yen H et al. Hydrological processes and model representation: Impact of soft data on calibration. Transactions of the Asabe, 2015, 58(6) : 1637-1660. DOI: 10.13031/trans.58.10726.

[97] Yen H, Bailey RT, Arabi M et al. The role of interior watershed processes in improving parameter estimation and performance of watershed models. Journal of Environmental Quality, 2014, 43(5) : 1601-1613. DOI: 10.2134/jeq2013.03.0110.

[98] Ding YL. Hydrological changes driven flood disaster simulation method[Dissertation]. Wuhan: Wuhan University, 2014. [丁雨淋. 水文变化驱动的暴雨-洪涝灾害主动模拟方法 [学位论文]. 武汉: 武汉大学, 2014.]

[99] Jung JW, Lim SS, Kwak JH et al. Further understanding of the impacts of rainfall and agricultural management practices on nutrient loss from rice paddies in a monsoon area. Water Air and Soil Pollution, 2015, 226(9) : 283. DOI: 10.1007/ s11270-015-2551-y.

[100] Yang S, Peng S, Xu J et al. Effects of water saving irrigation and controlled release nitrogen fertilizer managements on ni- 
trogen losses from paddy fields. Paddy and Water Environment, 2015, 13(1) : 71-80. DOI: 10.1007/s10333-013-0408-9.

[101] Yan R, Gao J, Huang J. WALRUS-paddy model for simulating the hydrological processes of lowland polders with paddy fields and pumping stations. Agricultural Water Management, 2016, 169: 148-161. DOI: 10.1016/j.agwat.2016.02.018.

[102] Xie X, Cui Y. Development and test of SWAT for modeling hydrological processes in irrigation districts with paddy rice. Journal of Hydrology, 2011, 396(1/2): 61-71. DOI: 10.1016/j.jhydrol.2010.10.032.

[103] Peng SZ, Xiong YJ, Luo YF et al. The effect of paddy field eco-ditch and wetland system on nitrogen and phosphorus pollutants reduction in drainage. Journal of Hydraulic Engineering, 2013, 44(6) : 657-663. [彭世彰, 熊玉江, 罗玉峰等. 稻田与沟塘湿地协同原位削减排水中氮磷的效果. 水利学报, 2013, 44(6):657-663.]

[104] Liu W, Yang H, Liu J et al. Global assessment of nitrogen losses and trade-offs with yields from major crop cultivations. Science of the Total Environment, 2016, 572: 526-537. DOI: 10.1016/j.scitotenv.2016.08.093.

[105] Garcia-Alba J, Barcena JF, Ugarteburu C et al. Artificial neural networks as emulators of process-based models to analyse bathing water quality in estuaries. Water Research, 2019, 150(MAR.1) : 283-295. DOI: 10.1016/j.watres.2018.11.063.

[106] Dazzi S, Vacondio R, Mignosa P. Integration of a levee breach erosion model in a GPU- accelerated 2D shallow water equations code. Water Resources Research, 2019, 55: 682-702. DOI: 10.1029/2018WR023826.

[107] Kim DH, Jang T, Hwang S. Evaluating impacts of climate change on hydrology and total nitrogen loads using coupled APEX-paddy and SWAT models. Paddy and Water Environment, 2020, 18(3) : 515-529. DOI: 10.1007/s10333-02000798-4.

[108] Rode M, Arhonditsis G, Balin D et al. New challenges in integrated water quality modelling. Hydrological Processes, 2010, 24(24) : 3447-3461. DOI; 10.1002/hyp.7766.

[109] Mohamoud Y, Zhang H. Applications of linked and nonlinked complex models for TMDL Development: Approaches and challenges. Journal of Hydrologic Engineering, 2019, 24(1) : 04018055. DOI: 10.1061/( asce) he.1943-5584.0001721. 


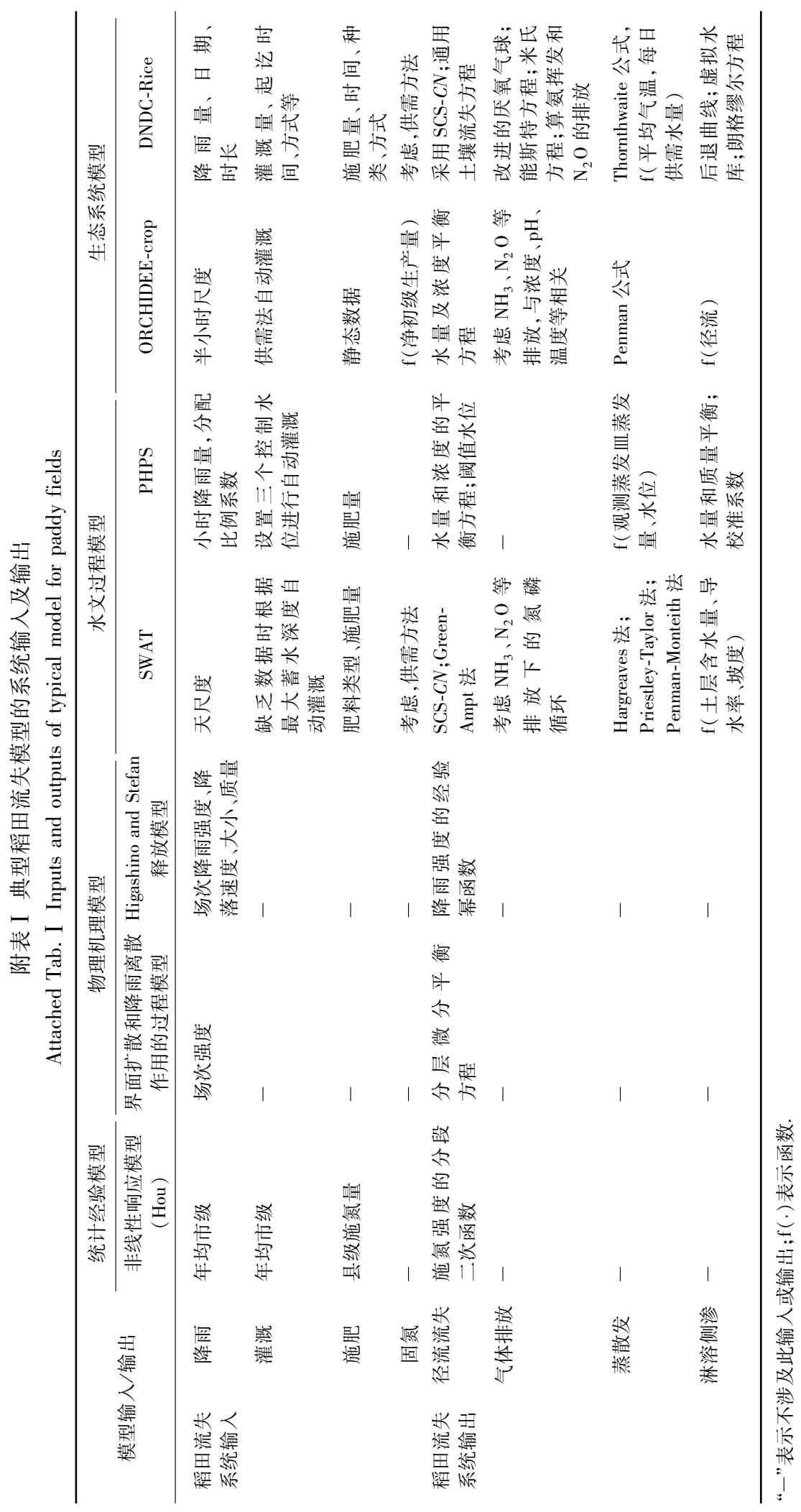




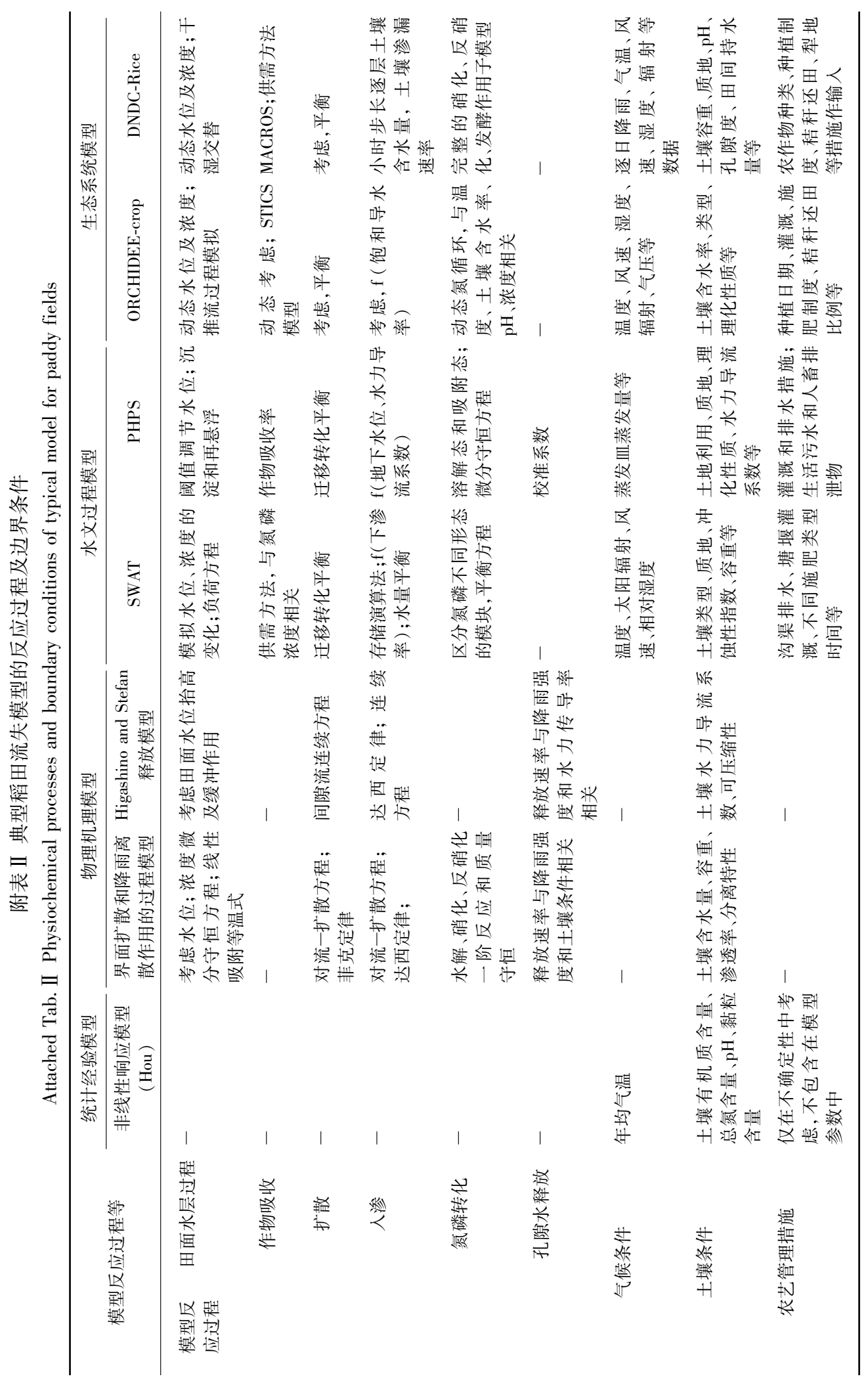

